\title{
Improved Message Delivery Scheme for UAVs Tracking the Moving Target
}

\author{
Yunlong $\mathrm{Yu}^{\mathrm{a}}$, Le Ru $\mathrm{Ru}^{\mathrm{b}}$ \\ Aeronautics and Astronautics Engineering College, Air Force Engineering University, Xi'an 710038, \\ China \\ ayuyunlong426@126.com, brule666@126.com
}

Keywords: ARIMA-WNN, UAV, message delivery scheme, moving target.

\begin{abstract}
Aiming at the special circumstance in which UAVs swarm are used in the mode of battlefield extending, a message delivery scheme called AWJPMMD (ARIMA-WNN Joint Prediction Model based Message Delivery) is proposed. In this scheme, the LET (Link Expiration Time) of the center node and the proxy node is calculated by high precision GPS information, then the LET at next moment is predicted by ARIMA-WNN (Autoregressive Integrated Moving Average model - Wavelet Neural Network) Joint Prediction Model. Finally, the process of message delivery is affected by the predicted value of LET and other parameters. The target information is sent to the UAVs ground station in form of store-and-forward by the message delivery process. Simulation shows that this scheme can provide higher message delivery ratio and this scheme is more stable.
\end{abstract}

\section{Introduction}

As we all know, UAVs (unmanned aerial vehicles) can carry out reconnaissance surveillance of single targets, so as to obtain intelligence data for the rear ground station [1-4]. When the distance between the rear UAV ground station and the target exceeds the maximum communication radius of the UAV, a common coping method is to construct a multi-UAV relay communication network to transmit reconnaissance data.

However, there is a situation in reality that the target is found by the UAV formation when it is too far from the UAV ground station, or because the target movement makes it too large for the UAV ground station. UAV clusters form the largest relay chain network (the distance between nodes and nodes is already the maximum communication radius) still cannot reach the target. If the unmanned aerial vehicle cluster is forced to form a linear formation between the target and the ground station (the distance between nodes exceeds the maximum communication radius), the effective communication of each node cannot be guaranteed, resulting in the fact that the reconnaissance surveillance data cannot be returned to the ground station, It is very detrimental to the rear decision.

Therefore, we propose a message delivery scheme AWJPMMD(ARIMA-WNN Joint Prediction Model based Message Delivery) based on ARIMA-WNN joint forecasting model. The AWJPMMD strategy is based on the ESF (Enhanced Store-and-Forward) algorithm and is a storage-forwarding message delivery strategy. Since the target is mobile and the location is unpredictable, the UAV cluster performs the search task first, and then performs the trace message delivery task. The AWJPMMD strategy enables effective reconnaissance of the target in the event that the target is too far from the UAV ground station, effectively transmitting the reconnaissance surveillance information.

\section{The Search Process of UAV Cluster}

In this paper, HELLO messages will be used to identify information about adjacent UAVs. The HELLO message contains the ID of the current UAV and the information provided by the current UAV GPS, and each UAV can broadcast the message periodically. The data packet mainly includes reconnaissance and monitoring of the target information and the GPS information of the central node. 
In order to achieve long-range target search and tracking, we assume that the UAV cluster in the search and tracking process to maintain a linear formation. In the search for a single target, the first discovered UAV will become the central node. Of course, the first to find the target may be more than one UAV, in order to quickly determine the center node and complete the search process, we use the UAV cluster single target search and node deployment algorithm, which is proposed by the literature [5].

\section{AWJPMMD Scheme}

In the single-target tracking process, the central node must maintain a certain distance from the target in order to effectively track the target and capture the target information, we assume that as long as the target in the central node communication range, the central node will be able to identify and obtain information. When the search process for the target is completed, the first UAV near the center node is treated as a proxy node, mainly for the forwarding of information. The proxy node and the central node can be kept within the communication range. At the same time, the proxy node should be as close as possible to the center node in order to monitor the location of the center node. The center node tracks the target in real time and is consistent with the speed of the target. The distance between the other nodes is equal, expressed as

$$
D=\frac{L-d}{N-1}
$$

Where $\mathrm{D}$ is the distance between the other nodes; $\mathrm{L}$ is the distance from the center node to the UAV ground station; $\mathrm{N}$ is the number of UAVs. Because the target is constantly moving, so $\mathrm{D}$ is not constant, each node can be through the UAV ground station and the central node of the current coordinate information, according to formula (1) to calculate the node should be in the location coordinates, dynamically changing the location of this node.

According to the hypothesis of this paper, the target is moving too far from the ground station, so that $\mathrm{D}$ is beyond the communication range of each node, reconnaissance information of the target cannot be returned to the ground station. In this paper, we first propose a LET (Link Expiration Time) estimation and ARIMA-WNN joint prediction model based on high-precision GPS information, and then integrate them into the algorithm to solve the problem effectively.

\subsection{Link Expiration Time Estimation Based on High Accuracy GPS Information}

Because GPS has positioning error (the error for the node position adjustment is tolerable), If you use the node location information in the HELLO message to estimate the LET between the central node and the agent node, it is very easy to introduce the positioning error, which affects the effective execution of the subsequent message delivery strategy.

In [6], the relative moving speed between the aircraft nodes is calculated by using the Doppler shift of the two continuously transmitted HELLO messages, and finally the LET is estimated. Although the method of [6] can estimate the more accurate LET, but in its message delivery strategy, the indicators used not only LET, as well as the central node in a HELLO message cycle movement distance $J_{M}$ (The distance is the absolute distance, need to use the GPS information). $J_{M}$ can introduce GPS positioning errors into it, adversely affecting message delivery strategies.

The use of high-precision GPS location information is an effective way to solve these problems. And the LET between the central node and the agent node can also be calculated with high accuracy GPS information. We use data fusion method to produce high-precision GPS information. Data fusion mainly uses the extended Kalman filter EKF, the specific operation process is described in [7-9].

The process of calculating the LET between the central node and the proxy node using high precision GPS information is

$$
L E T=\frac{-(a b+c d)+\sqrt{\left(a^{2}+c^{2}\right) r^{2}-(a d-b c)^{2}}}{a^{2}+c^{2}}
$$




$$
\begin{aligned}
& a=v_{i} \cos \theta_{i}-v_{j} \cos \theta_{j} \\
& b=x_{i}-x_{j} \\
& c=v_{i} \sin \theta_{i}-v_{j} \sin \theta_{j} \\
& d=y_{i}-y_{j}
\end{aligned}
$$

\subsection{ARIMA-WNN Joint Forecasting Model}

The estimation of the LET is only an estimate of the current time. If a certain prediction mechanism can be used to predict the LET at the next time, the whole UAV cluster network will have some predictability.

Firstly, the linear part of LET time series is modeled by ARIMA model, and the prediction result $\hat{L}_{t}$ is obtained. The residual of the predicted result and the original LET time series implies the non-linearity of the LET time series. Then, this part of the residuals are substituted into the WNN model. The model predicts the nonlinear characteristics of the LET time series by constantly correcting the connection weights and thresholds between the neurons and predicts the results $\hat{U}_{t}$.

Finally, the two parts of the forecast results are added, that is, the next time LÊT .

\subsection{Message Delivery Scheme}

In this strategy, the central node is mainly responsible for tracking the target, need to keep a close distance with the target and the speed is consistent to the target. The central node broadcasts HELLO messages at certain intervals. The central node transmits the currently acquired target information, and the target information is sent in a certain period and is updated in real time.

The proxy node is the sender of the central node information, unlike any other node. The proxy node needs to be close to the central node in order to indirectly track the target. In this paper, the distance between the two nodes is set at $10 \mathrm{~m}$. When the proxy node receives the HELLO message of the central node, the node will calculate the LET according to the high precision GPS information and use the ARIMA-WNN model to predict the LET at the next time. When LÊT is greater than a constant, the link is considered stable, and the message can be delivered. At the same time, other nodes in the message delivery process will adjust the position of the node according to the coordinates of the center node to adapt to the target movement. On the contrary, when the link is in an unstable state, there are two strategies: When the center node moves in a message cycle more than a certain value, then it means that the target movement speed is too fast, we need to immediately adjust the location of all nodes, while sending the information to the station; On the contrary, the target movement is not very fast, then only need to adjust the location of the agent node, in a short time to restore the link stability, so as to restore the message delivery. When the proxy node returns, it only needs to return to the original coordinates.

The other node judges whether it is the recipient of the information or the sender according to the HELLO message and reliably accepts or transmits the target information.

\section{Simulations}

In the simulation scenario, 6 to $10 \mathrm{UAVs}$ are located in the $4000 \mathrm{~m} * 4000 \mathrm{~m}$ area, the initial coordinates of the target is $(0,2000)$, the minimum speed is $0.1 \mathrm{~m} / \mathrm{s}$, the maximum speed is $7 \mathrm{~m} / \mathrm{s}$. The direction of movement of the target is random, but its scope of activity is limited to $300 \mathrm{~m}$. The coordinate of the UAV ground station is $(0,0)$. The maximum speed of all UAVs is limited to 15,20 , and $25 \mathrm{~m} / \mathrm{s}$, respectively, with a maximum communication radius of $200 \mathrm{~m}$. Each simulation time is 600 s, a total of 30 simulations, all the results are the average of the simulation. The simulation software used in this paper is NS-2.

In this paper, we select the SF algorithm [5], ESF algorithm [6], RRS algorithm [2] and AWJPMMD strategy to compare the message delivery ratio.

Figure 1 shows the difference in the ratio of message delivery among the four strategies in different time periods under $10 \mathrm{UAVs}$ and their moving speed is $20 \mathrm{~m} / \mathrm{s}$. The message delivery ratio of the 
AWJPMMD strategy is higher than that of the other three algorithms over the entire time period, which fully demonstrates the stability of the strategy in terms of message delivery ratio.

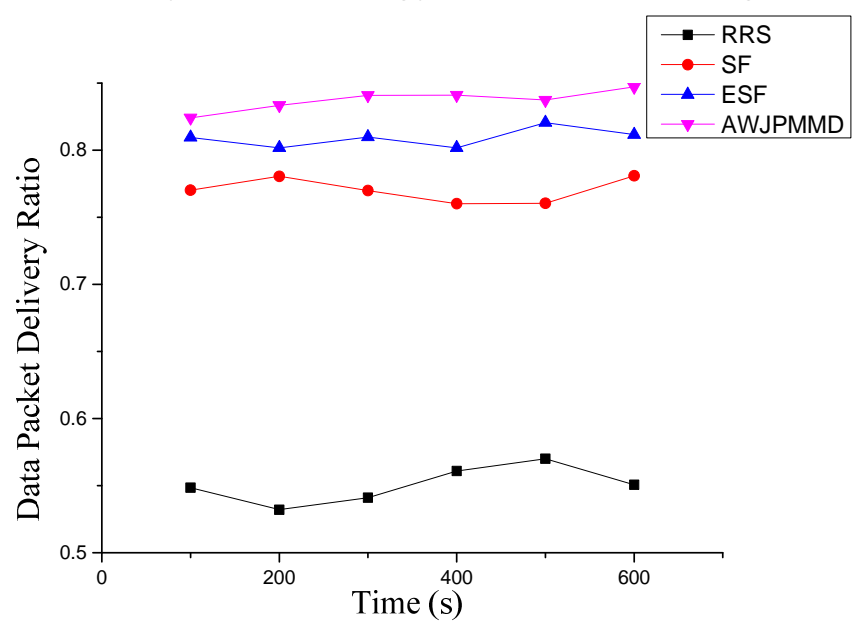

Figure 1 Comparison of data packet delivery ratio at different times

Figure 2 shows the difference in the ratio of message delivery among the four strategies in different UAV number and their moving speed is $20 \mathrm{~m} / \mathrm{s}$. The AWJPMMD strategy maintains a high message delivery success rate in the number of different UAV deployments, which is inseparable from the joint forecasting mechanism of the AWJPMMD strategy. From a simple algorithm, increasing the number of UAV deployments can improve the success rate of message delivery.

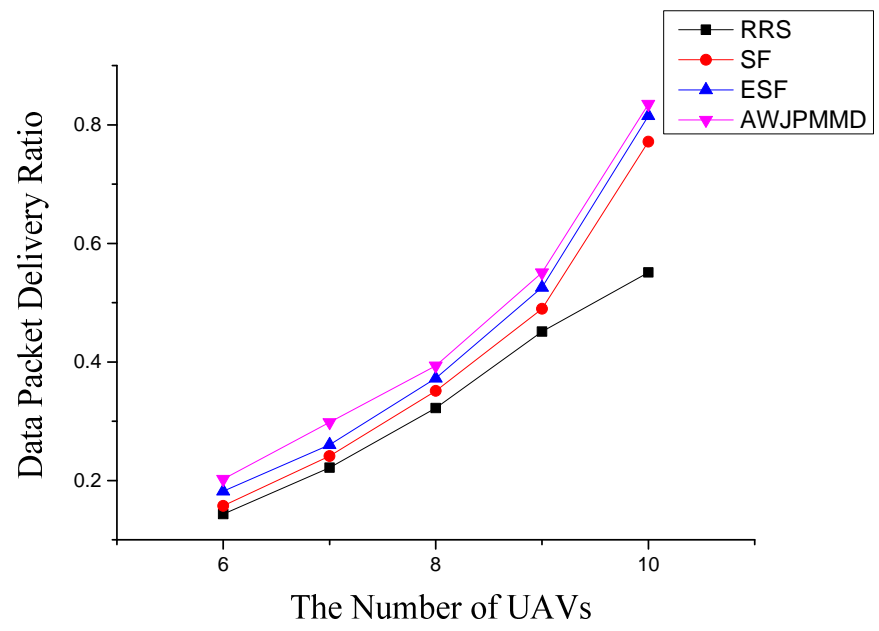

Figure 2 Comparison of the data packet delivery ratio for different UAV numbers

\section{Conclusion}

AWJPMMD scheme first calculates the LET between the central node and the proxy node according to the high precision GPS information, and then predicts the next time the LET according to the ARIMA-WNN joint prediction model. Finally, carrying out the message delivery process according to the predicted value of LET and other parameters, so that the target information is transmitted to the UAV ground station in the form of storage - forwarding. The simulation results show that the AWJPMMD scheme can provide higher data packet delivery ratio and better stability than the other three schemes. 


\section{References}

[1] Cheng C M. Maximizing throughput of UAV-relaying networks with the load-carry-and-deliver paradigm [C]// in Proceedings of International Conference on Communications. Geneva, Switzerland: IEEE Press, 2007. 4417-4424.

[2] Niklas G, Kai D, Christian W. Role-Based Connectivity Management with Realistic Air-to-Ground Channels for Cooperative UAVs [J]. IEEE Journal on Selected Areas in Communications, 2012, 30(5): 951-963.

[3] M. Grossglauser, D. N. C. Tse. Mobility increases the capacity of ad hoc wireless networks [J]. IEEE/ACM Transactions on Networking, 2002, 10(4).

[4] D. Henkel, T. Brown. Delay-tolerant communication using mobile robotic helper nodes in Modeling and Optimization in Mobile Ad Hoc and Wireless Networks and Workshops [C]// in WiOPT 2008 6th International Symposium on, April 2008. 657 -666

[5] Hyo H C, Su H N, Taeshik S, et al. Information delivery scheme of micro UAVs having limited communication range during tracking the moving target [J]. J Supercomput, 2013, 66(10): 950-972.

[6] Yu Yunlong, Ru Le. Information delivery algorithm for UAVs tracking remote moving target [J]. Application research of computers, 2016, 33(9): 2625-2629.

[7] M. S. Grewal, A. P. Andrews. Kalman Filtering Theory and Practice. Englewood Cliffs, NJ: PRENTICE-Hall, 1993.

[8] Yu Yunlong, Ru Le. A method of locating light UAV and correcting heading [J]. computer simulation, 2015, 32(11): 71-74.

[9] Nima Alam, Asghar Tabatabaei Balaei. A DSRC Doppler-Based Cooperative Positioning Enhancement for Vehicular Networks with GPS Availability [J]. IEEE Transaction on vehicular technology, 2011, 60(9): 4462-4470. 